\title{
Examining the Human Element in Lab Biosafety
}

\author{
Betiel H. Haile ${ }^{1}$, Mark Wade ${ }^{2}$, Patricia Blevins ${ }^{2}$, Andrew Cannons ${ }^{3}$, Richard France ${ }^{3}$, Lisa \\ D. Ferland ${ }^{1}$, Affan Shaikh ${ }^{1}$, Meeyoung Park ${ }^{\star 1}$, Ngozi Erondu', Sean G. Kaufman ${ }^{4}$, Heather \\ Meeks ${ }^{5}$ and Scott J. McNabb ${ }^{1,4}$
}

${ }^{1}$ Public Health Practice, LLC, Atlanta, GA, USA; ${ }^{2}$ San Antonio Metropolitan Health District, San Antonio, TX, USA; ${ }^{3}$ Bureau of Public Health Laboratories-Tampa, Tampa, FL, USA; 'Emory University, Atlanta, GA, USA; ${ }^{5}$ Defense Threat Reduction Agency, Ft. Belvoir, VA, USA

\section{Objective}

To understand the potential gaps in laboratory biosafety due to human factors.

\section{Introduction}

A laboratory biosafety program (LBP) is essential to ensure the health and safety of laboratory staff and the general public from hazardous materials and infectious agents. In the US, the Occupational Safety and Health Administration (OSHA) sets federal standards governing LBPs that enforce best practices by non-regulatory organizations such as the Centers for Disease Control and Prevention (CDC) and the National Institutes of Health (NIH) [1]. In addition, twenty-five states and two territories established OSHA-approved biosafety standards that meet or exceed federal standards [2]. The CDC/NIH's Biosafety in Microbiological and Biomedical Laboratories $[\mathrm{BMBL}]$ are the primary guidelines for LBPs, and many jurisdictions use the manual in a regulatory manner [3]. Ensuring laboratory biosafety requires vigilance; laboratories must maintain equipment and materials, develop and implement security measures, and staff must be annually trained in biosafety procedures. Our evaluation of LBPs underscored the importance of the human element in biosafety compliance.

\section{Methods}

We conducted a literature review on LBPs to identify objectives, goals, and best practices. Findings were organized in a logic model to assess performance in biosafety, biosurety, protocols, physical security, information security, and training. Indicators were identified in existing literature (e.g., CDC Inspection Checklist for BSL-2, BMBL v5) or developed and mapped to the logic model $[3,4]$.

Field tests of the evaluation tool were conducted at the San Antonio Metropolitan Health District Public Health Laboratory and the Bureau of Public Health Laboratories in Tampa, Florida. Eleven staff, including a lab director, lab coordinator, lab technologists, and lab technicians, was interviewed at the San Antonio site. Twenty-five staff, including supervisors, microbiologists, medical scientists, lab technicians, virologists, serologist, and administrative staff, was interviewed at the Tampa site. We evaluated laboratory area biosafety levels (BSL) 1, 2 and 3 at both sites.

\section{Results}

The tool included 130 indicators administered in three formats: pre-visit questionnaire (58), on-site observation checklist (56), and staff interview (16). Pre-visit questions and on-site observations determined that both sites fully implemented a LBP. Staff interviews elicited a range of responses on the efficacy of biosafety training. Using averaged Likert scale ratings, both sites were considered "very capable" at operating safely with biologic materials and "very effective" at reducing or controlling biologic exposures. The BSL-3 staff was rated "very competent" to "extremely competent at donning respirators and Tyvek suits. Reasons stated for lower ratings included unfamiliarity with under-utilized protocols and equipment, lack of more engaging biosafety training, and complacency/human error (at least one person stated that "nobody is perfect").

\section{Conclusions}

In any laboratory, a proper LBP is a critical component of laboratory practice. Nonetheless, specific funding for laboratories to conduct biosafety trainings and engage the workforce on biosafety is often overlooked and, therefore, opportunities to develop or acquire biosafety training materials are extremely limited or non-existent. Staff would benefit from various training formats (e.g., hands-on, small group) to accommodate different learning styles. Additional refresher trainings are recommended for non-routine protocols and non-technical staff passing through biosafety risk areas. Finally, a third-party, or confidential assessment of laboratory staff, is necessary to collect unbiased opinions on a LBP to implement better interventions.

\section{Keywords}

biosafety; laboratory; assessment

\section{Acknowledgments}

Scott Becker (APHL)

Chris Mangal (APHL)

\section{References}

1. OSHA. Laboratory Safety Guidance [Internet]. 2011 [cited 2013 Sept 2] Available from https://www.osha.gov/Publications/laboratory/OSHA3404laboratory-safety-guidance.pdf

2. OSHA. State Occupational Safety and Health Plans [Internet]. Washington: US Department of Labor; n.d. [cited 2013 Sept 2]. Available from https://www.osha.gov/dcsp/osp/index.html

3. CDC, NIH. Biosafety in Microbiological and Biomedical Laboratories. 5th edition. In: Chosewood L, Wilson D. (eds.). Washington: US Government Printing Office; 2009.

4. APHIS/CDC Select Agent Program. Inspection Checklist for BSL-2 Laboratories. Washington; 2011.

*Meeyoung Park
E-mail: mpark@publichealthpractice.com 